\title{
Effect of sirolimus on malignancy and survival after kidney transplantation: systematic review and meta-analysis of individual patient data
}

\author{
(c) (1) (2) OPEN ACCESS
}

\begin{abstract}
Greg A Knoll nephrologist ${ }^{12}$, Madzouka B Kokolo research coordinator ${ }^{1}$, Ranjeeta Mallick statistician ${ }^{1}$, Andrew Beck research assistant ${ }^{1}$, Chieny D Buenaventura research assistant ${ }^{1}$, Robin Ducharme methodologist $^{12}$, Rashad Barsoum professor ${ }^{3}$, Corrado Bernasconi medical director ${ }^{4}$, Tom D Blydt-Hansen nephrologist ${ }^{5}$, Henrik Ekberg professor ${ }^{6}$, Claudia R Felipe transplant coordinator ${ }^{7}$, John Firth consultant nephrologist ${ }^{8}$, Lorenzo Gallon professor of medicine ${ }^{9}$, Marielle Gelens nephrologist ${ }^{10}$, Denis Glotz nephrologist ${ }^{11}$, Jan Gossmann nephrologist ${ }^{12}$, Markus Guba professor of surgery $^{13}$, Ahmed Ali Morsy professor of urology ${ }^{14}$, Rebekka Salgo consultant dermatologist ${ }^{15}$, Earnst H Scheuermann nephrologist ${ }^{12}$, Helio Tedesco-Silva nephrologist ${ }^{7}$, Stefan Vitko nephrologists ${ }^{16}$, Christopher Watson professor of transplantation ${ }^{17}$, Dean A Fergusson epidemiologist $^{12}$
\end{abstract}

\begin{abstract}
'Ottawa Hospital Research Institute, Ottawa, ON, Canada; ${ }^{2}$ University of Ottawa, Ottawa, ON, Canada; ${ }^{3}$ Cairo Kidney Center, Cairo University, Cairo, Egypt; ${ }^{4}$ Limites Medical Research, Vacallo Switzerland; ${ }^{5}$ University of Manitoba, Department of Pediatrics and Child's Health, Winnipeg, MB, Canada; ${ }^{6}$ Department of Nephrology and Transplantation, Lund University, Malmo, Sweden; ${ }^{7}$ Hospital do Rim e Hipertensao, Universidade Federal de Sao Paulo, Sao Paulo, Brazil; ${ }^{8}$ Department of Renal Medicine, Addenbrooke's Hospital, Cambridge, UK; ${ }^{9}$ Northwestern University, Feinberg School of Medicine, Chicago, IL, USA; ${ }^{10}$ Maastricht University Medical Centre, Maastricht, Netherlands; ${ }^{11}$ Department of Nephrology, Saint-Louis Hospital, Paris, France; ${ }^{12}$ Division of Nephrology, Hospital of J.W. Goethe, Frankfurt, Germany; ${ }^{13}$ Department of Surgery, University of Munich, Munich, Germany; ${ }^{14}$ Cairo Kidney Center, Cairo University, Cairo, Egypt; ${ }^{15} \mathrm{Clinic}$ of Dermatology, Venerology and Allergology, JW Goethe Clinic, University of Frankfurt, Germany; ${ }^{16}$ Department of Nephrology, Institutu Klinicke a Experimentalni Mediciny, Prague, Czech Republic; ${ }^{17}$ University of Cambridge, Department of Surgery, NIHR Cambridge Biomedical Research Centre, Addenbrooke's Hospital, Cambridge, UK
\end{abstract}

\begin{abstract}
Objective To examine risk of malignancy and death in patients with kidney transplant who receive the immunosuppressive drug sirolimus.

Design Systematic review and meta-analysis of individual patient data.

Data sources Medline, Embase, and the Cochrane Central Register of Controlled Trials from inception to March 2013.

Eligibility Randomized controlled trials comparing immunosuppressive regimens with and without sirolimus in recipients of kidney or combined pancreatic and renal transplant for which the author was willing to provide individual patient level data. Two reviewers independently screened titles/abstracts and full text reports of potentially eligible trials to identify studies for inclusion. All eligible trials reported data on malignancy or survival.
\end{abstract}

Results The search yielded 2365 unique citations. Patient level data were available from 5876 patients from 21 randomized trials. Sirolimus was associated with a $40 \%$ reduction in the risk of malignancy (adjusted hazard ratio $0.60,95 \%$ confidence interval 0.39 to 0.93 ) and a $56 \%$ reduction in the risk of non-melanoma skin cancer $(0.44,0.30$ to 0.63$)$ compared with controls. The most pronounced effect was seen in patients who converted to sirolimus from an established immunosuppressive regimen, resulting in a reduction in risk of malignancy $(0.34,0.28$ to $0.41)$, non-melanoma skin cancer $(0.32,0.24$ to 0.42$)$, and other cancers $(0.52,0.38$ to 0.69$)$. Sirolimus was associated with an increased risk of death $(1.43,1.21$ to 1.71$)$ compared with controls.

Conclusions Sirolimus was associated with a reduction in the risk of malignancy and non-melanoma skin cancer in transplant recipients. The 
benefit was most pronounced in patients who converted from an established immunosuppressive regimen to sirolimus. Given the risk of mortality, however, the use of this drug does not seem warranted for most patients with kidney transplant. Further research is needed to determine if different populations, such as those at high risk of cancer, might benefit from sirolimus.

\section{Introduction}

Kidney transplantation is the treatment of choice for people with end stage renal disease as it improves survival ${ }^{1}$ and quality of life compared with dialysis. ${ }^{2}$ Success in transplantation has occurred to a great extent by the introduction of more potent immunosuppressive drugs. These drugs have reduced the incidence of acute rejection from 50\% in 1995 to less than $10 \%$ today. ${ }^{3}$ In the United States alone there are more than 185000 patients alive with a functioning kidney transplant; a number that has more than doubled since $1995 .{ }^{3}$ Similar improvement has been reported in Europe, Asia, and Australia. ${ }^{4}$ The downside of this success is that more patients are at risk of

immunosuppressive side effects related to the drugs, with the most serious being malignancy. ${ }^{5}$ The incidence of most cancers increases substantially after kidney transplantation. ${ }^{56}$ In addition to substantial patient morbidity, cancer accounts for $10-30 \%$ of all deaths after transplant. ${ }^{78}$ Non-melanoma skin cancers (basal and squamous cell carcinoma) are by far the most common type of cancer, occurring in up to a third of kidney transplant recipients. ${ }^{9}$ Unlike in the general population, squamous cell skin cancers can be quite aggressive in transplant recipients, with a high recurrence rate, metastasis, and death. ${ }^{10-12}$ Reduction in the incidence and complications associated with malignancy would be a major advance for kidney transplant recipients.

Sirolimus, a mammalian target of rapamycin (mTOR) inhibitor, is an immunosuppressive drug used in kidney transplantation. The mTOR pathway is a key regulator of cell growth and survival, and this pathway is often dysregulated in many types of malignancy. ${ }^{13}$ As such there has been interest in the use of sirolimus to reduce the risk of cancer. ${ }^{14-16}$ An observational study of the early experience with mTOR inhibitors found a $60 \%$ reduction in cancers for patients receiving an mTOR inhibitor compared with those who received a calcineurin inhibitor. ${ }^{16}$ Results from randomized trials have not been as convincing. A meta-analysis published by the Cochrane Renal Group in 2006 showed a non-significant trend toward a lower risk of malignancy for patients treated with sirolimus. ${ }^{17}$ Trials published since then have produced conflicting results. Two small trials have both shown a significant reduction in skin cancer after conversion from a calcineurin inhibitor to sirolimus, but neither showed a reduction in other types of malignancy. ${ }^{18}{ }^{19}$ The SYMPHONY study, one of the largest transplant trials, failed to show a reduction in cancer for those randomized to sirolimus. ${ }^{20}$ Intuitively, the reduction in cancer with sirolimus should also lead to improved survival. The Cochrane meta-analysis showed a possible trend towards improved survival for patients treated with sirolimus compared with those receiving a calcineurin inhibitor. ${ }^{17}$ This analysis, however, was underpowered as they had data on only 631 patients from six trials. ${ }^{17}$

We investigated the effect of sirolimus on development of cancer and on survival among transplant recipients using data from randomized trials. We hypothesized that sirolimus would be associated with a significant reduction in both malignancy and death.

\section{Methods \\ Pooled studies}

We conducted a systematic review to identify all randomized trials comparing immunosuppressive regimens with and without sirolimus in recipients of kidney or combined pancreatic and renal transplant. To be eligible, trials needed to collect data on malignancy after transplantation and have a planned follow-up period of at least three months. We searched Medline, Embase, and the Cochrane Central Register of Controlled Trials from inception to 19 March 2013. Two trained reviewers independently screened titles/abstracts and full text reports of potentially eligible trials to identify studies for inclusion. The corresponding author of each eligible trial was contacted and asked to provide individual patient data. All initial communications with authors were based on a template explaining the study and the data required. Two separate reminders were sent two weeks apart unless we had received a definitive response. Several trial datasets were provided directly by Pfizer (previously Wyeth Pharmaceuticals).

\section{Data and variables}

From each author, we requested an anonymized dataset describing study characteristics, patient characteristics, transplantation details, and events recorded during the trial. We classified malignancies as non-melanoma skin cancer (basal cell or squamous cell carcinoma of the skin) or other cancer (all other malignancies). When more than one type of malignancy was recorded on the same day in the same patient, each type was coded individually. We classified deaths by cause: cardiovascular, infection, malignancy, respiratory, gastrointestinal, sudden death, other, or unknown. The reviewers determined classification of occurrence of malignancy and type as well as cause of death without knowledge of treatment assignment. The pooled data were validated against the original trial publications for accuracy. We assessed study quality with the Cochrane Collaboration's tool for assessing risk of bias and publication bias with funnel plots.

\section{Study outcomes}

The primary outcome was the development of any new malignancy after randomization. This outcome included any type of cancer (such as basal cell skin cancer, lymphoma, etc). Secondary outcomes included non-melanoma skin cancer, other cancer, and death. We did not consider any events recorded outside of the context of the original trial (for example, during an observational follow-up extension study). As a sensitivity analysis we analyzed, in aggregate form, the pooled risk of malignancy, non-melanoma skin cancer, and death from all trials identified as eligible for inclusion. This included the trials for which we obtained individual patient level data as well as the trials for which we had only trial level aggregate data.

\section{Statistical analyses}

We used a modified intention to treat approach. All participants were analyzed according to the group to which they were originally randomized. We excluded randomized patients who did not receive the study drug or did not undergo transplantation. The primary analysis estimated the time to any first malignancy. Similar analyses were performed for the secondary outcomes: times to first non-melanoma skin cancer, to other cancer, and to death. We pooled the individual patient level data and used Kaplan-Meier curves to calculate survival. Cox proportional hazards models were used to determine adjusted hazard ratios 
with $95 \%$ confidence intervals. All Cox models were adjusted for trial, age, sex, geographic location of participant, and time after transplantation to randomization, with individual studies considered as having random effects. For analyses of time to malignancy, participants were censored at death or the date of last follow-up. For patient survival, participants were censored at date of last follow-up. Given that trials had differing lengths of follow-up, we performed a sensitivity analysis that censored all participants at 24 months. Subgroup analyses were performed to evaluate the effect of sirolimus by trial design and drug exposure. We examined conversion trials (in which sirolimus replaced another immunosuppressive drug, most often a calcineurin inhibitor, at some time point after transplantation) separately from de novo trials (sirolimus started as the initial treatment at the time of transplantation). We did not have patient level data on sirolimus drug concentration so we used the average drug concentration achieved or target drug concentration from published trial reports to categorize studies as low dose or high dose trials (below or above the median sirolimus drug concentration of $10 \mathrm{ng} / \mathrm{mL}$ ). We performed supplementary subgroup analyses to examine the effect of sirolimus by type of donor (living $v$ dead) and the use of antibody induction therapy (yes $v$ no). Donor type and antibody induction therapy were subgroups within a trial and not trial level characteristics. We compared survival curves with the log rank test and proportions with $\chi^{2}$ tests. We calculated the number needed to treat and the number needed to harm for time to event data. ${ }^{21}$ All reported $\mathrm{P}$ values are two sided.

\section{Results}

\section{Characteristics of the participants}

We included 21 trials ${ }^{18-40}$ (6894 participants) and received individual patient data for 5963 patients (two trials were unable to provide individual patient data for 931 participants $^{38}{ }^{39}$ ). None of the 21 trials included patients who had received a combined pancreatic and renal transplant. Additionally, 87 participants were excluded as they never received a transplant or did not receive any study drug, leaving 5876 eligible patients. Figure $1 \Downarrow$ outlines the search and selection of randomized controlled trials for the review. Table $1 \Downarrow$ shows the characteristics of the patients. In the control group, only 156 patients received placebo and the remaining 2444 received an active drug comparator. The patients randomized to placebo also received cyclosporine and prednisone. Table A in appendix 1 describes the features of the 21 included trials including target drug concentrations. Table B in appendix 1 outlines the risk of bias for each trial, and figure $\mathrm{A}$ in appendix 2 shows the funnel plots for the outcomes of cancer and death.

\section{Primary analysis}

In total 243 patients developed a malignancy: 127 in the sirolimus group and 116 in the control group. The cumulative incidence of cancer was lower in the sirolimus group than in the control group ( $\mathrm{P}=0.006$; fig $2 \Downarrow)$. After multivariable adjustment, the reduction in the risk of malignancy remained significant (adjusted hazard ratio $0.60,95 \%$ confidence interval 0.39 to $0.93 ; \mathrm{P}=0.02)($ table $2 \Downarrow)$. Each one year increment in age was associated with a $6 \%$ increase risk of cancer $(1.06,1.04$ to $1.09 ; \mathrm{P}<0.001$ ) (table 2 ). There was a $20 \%$ reduction in risk of malignancy in women compared with men $(0.80,0.66$ to $0.97 ; \mathrm{P}=0.03$ ) (table 2).

Geographic location of patient was associated with cancer. Compared with patients in North America, those in Oceania had a threefold increased risk of malignancy $(3.68,2.21$ to 6.13 ;
$\mathrm{P}<0.001$ ), while those living in Africa (0.31, 0.23 to 0.41; $\mathrm{P}<0.001)$, Asia (0.87, 0.58 to $1.30 ; \mathrm{P}=0.49)$, Europe $(0.58,0.44$ to $0.76 ; \mathrm{P}<0.001)$, and South America $(0.59,0.42$ to 0.85 ; $\mathrm{P}=0.004$ ) had a lower risk of cancer (table $2 \Downarrow$ ). Overall, the causes of cancer were significantly different in the sirolimus and control groups $(\mathrm{P}=0.004$; table $\mathrm{C}$ in appendix 1$)$. There was a higher proportion of squamous $(2.0 \% v 1.3 \%)$ and basal $(1.4 \%$ $v 0.79 \%$ ) cell skin cancer in the control group compared with the sirolimus group, while there were more hematological malignancies in the sirolimus group $(0.64 \% v 0.19 \%)$.

\section{Secondary analyses}

The cumulative incidence of non-melanoma skin cancer was significantly greater in the control arm than in the sirolimus group $(\mathrm{P}<0.001$; fig $3 \Downarrow)$. The reduction in non-melanoma skin cancer for those receiving sirolimus remained significant after multivariable adjustment (adjusted hazard ratio $0.44,95 \%$ confidence interval 0.30 to $0.63 ; \mathrm{P}<0.001)$. There was no significant difference in the occurrence of other cancers in those receiving sirolimus compared with control $(\mathrm{P}=0.65$; fig $\mathrm{B}$ in appendix 2). Multivariable adjustment did not change this association $(1.05,0.57$ to $1.94 ; \mathrm{P}=0.88)$. We carried out separate analyses for sirolimus compared with placebo and sirolimus compared with an active comparator, but the conclusions did not differ for the outcomes of any cancer, non-melanoma skin cancer, or other cancer (data not shown).

There were 202 deaths: 133 in the sirolimus group and 69 in the control group (table D in appendix 1 and fig $\mathrm{C}$ in appendix 2). Overall patient survival was significantly reduced in the sirolimus group $(\mathrm{P}=0.04$; fig $4 \Downarrow)$. After multivariable adjustment, sirolimus use was associated with a $43 \%$ increased risk of death compared with the control group (adjusted hazard ratio 1.43, 1.21 to $1.71 ; \mathrm{P}<0.001$ ) (table $2 \Downarrow$ ). The causes of death were significantly different in the two groups $(\mathrm{P}=0.002$; table $\mathrm{D}$ in appendix 1). There were relatively few deaths attributed to malignancy, but the proportions were similar in the two groups $(0.21 \%$ sirolimus, $0.19 \%$ control). There was a higher proportion of death from infection $(0.58 \% v 0.15 \%)$ and cardiovascular disease $(1.28 \% v 0.54 \%)$ in the sirolimus group compared with the control group. There was a trend towards improved graft survival after deaths were censored in the sirolimus patients $(\mathrm{P}=0.06$; fig $\mathrm{D}$ in appendix 2).

\section{Subgroup analyses}

In conversion trials, the cumulative incidence of cancer was lower in the sirolimus group than in the control group $(\mathrm{P}<0.001$; fig $\mathrm{E}$ in appendix 2), which persisted after adjustment for covariates. Patients receiving sirolimus had a $66 \%$ reduction in the risk of cancer (adjusted hazard ratio $0.34,95 \%$ confidence interval 0.28 to $0.41 ; \mathrm{P}<0.001$ ) (fig $5 \Downarrow$ ). Sirolimus use was associated with a $68 \%$ reduction in the risk of non-melanoma skin cancer $(0.32,0.24$ to $0.42 ; \mathrm{P}<0.001)$ and a $48 \%$ reduction in the risk of other cancers $(0.52,0.38$ to $0.69 ; \mathrm{P}<0.001)$. As with the overall analysis, sirolimus use was associated with an increased risk of death in the conversion trials $(1.59,1.36$ to 1.85 ; $\mathrm{P}<0.001$ ) (fig $5 \Downarrow$ ).

Patients in de novo trials showed different results with respect to occurrence of cancer. Sirolimus use was not associated with a reduced risk of cancer (adjusted hazard ratio 1.09, 95\% confidence interval 0.74 to $1.61 ; \mathrm{P}=0.65$ ) (fig $5 \Downarrow$ ), non-melanoma skin cancer $(0.65,0.36$ to $1.17 ; \mathrm{P}=0.15)$ (fig 5), or other cancers $(1.70,0.98$ to $2.93 ; \mathrm{P}=0.06)$. Consistent with the other analyses, sirolimus use was associated with a 
significant increase in the risk of death in de novo trials $(1.39$, 1.12 to $1.73 ; \mathrm{P}=0.003$ ) (fig 5).

In low dose sirolimus trials, there was no difference in the risk of malignancy (adjusted hazard ratio $0.65,95 \%$ confidence interval 0.30 to $1.41 ; \mathrm{P}=0.27$ ), but there was a significant reduction in non-melanoma skin cancer $(0.43,0.24$ to 0.78 ; $\mathrm{P}=0.006$ ) (fig $5 \Downarrow$ ) for patients taking sirolimus compared with control. There was no significant difference in the risk of death between the two groups $(1.07,0.81$ to $1.41 ; \mathrm{P}=0.65$ ) (fig $5 \Downarrow$ ). In high dose sirolimus trials, there was a significant reduction in the risk of malignancy $(0.57,0.36$ to $0.91 ; \mathrm{P}=0.02)$ and non-melanoma skin cancer $(0.43,0.26$ to $0.70 ; \mathrm{P}<0.001)$ (fig 5 ) for patients taking sirolimus compared with control. Sirolimus use was associated with an increased risk of death in the high dose sirolimus trials $(1.53,1.15$ to $2.05 ; \mathrm{P}=0.004)$ (fig 5).

For patients who did not receive antibody induction therapy, sirolimus use was associated with a non-significant reduction in any cancer (adjusted hazard ratio $0.68,95 \%$ confidence interval 0.45 to 1.01 ) and a significant reduction in non-melanoma skin cancer $(0.35,0.22$ to 0.55$)$ (fig $F$ in appendix 2). Sirolimus use was associated with a significant increase in other cancers $(1.82,1.25$ to 2.65$)$ and a null effect for death (1.02, 0.77 to 1.34 ) for those who did not receive induction therapy (fig $F$ in appendix 2). For the antibody induction group sirolimus use was associated with a non-significant increase in any cancer (1.26, 0.61 to 2.61$)$, non-melanoma skin cancer (1.42, 0.49 to 4.14$)$, and other cancers $(1.25,0.39$ to 3.98$)$, with a significant increase in death $(1.90,1.36$ to 2.68$)$ (fig $F$ in appendix 2).

For recipients of organs from both living and deceased donors, the findings were consistent with the overall results for the outcomes of any cancer, non-melanoma skin cancer, and other cancer (fig $F$ in appendix 2). For the outcome of death, sirolimus use was associated with an increased risk of death in recipients from deceased donors (adjusted hazard ratio 1.52, 95\% confidence interval 1.21 to 1.91 ), but there was a null effect in recipients with living donors $(1.00,0.56$ to 1.78$)$ (fig $\mathrm{F}$ in appendix 2).

\section{Sensitivity analysis}

As a sensitivity analysis we analyzed the trial level pooled risk of malignancy, non-melanoma skin cancer, and death from all trials identified as eligible for inclusion (table $3 \Downarrow$ ). Of those that reported only trial level data, 16 reported on cancer, nine reported on non-melanoma skin cancer, and 29 reported death (details of these trials are reported table $\mathrm{E}$ in appendix 2). The pooled relative risk combined was 0.63 ( $95 \%$ confidence interval 0.48 to 0.81 ) for any cancer and 0.47 (0.37 to 0.61$)$ for non-melanoma skin cancer (table $3 \Downarrow$ ). For the outcome of death, when all trials were pooled together regardless of duration of follow-up the relative risk was 1.21 (0.97 to 1.51 ). When we confined the analysis to trials with greater than 24 months of follow-up ( $n=11$ trials; $n=2507$ patients), sirolimus use was associated with a $42 \%$ increased risk of death compared with the control group (1.42, 1.03 to 1.96$)$.

Censoring of patients at 24 months did not have any material effect on the study outcomes (table $4 \Downarrow$ ). The use of sirolimus remained associated with a significant reduction in any cancer (adjusted hazard ratio $0.55,95 \%$ confidence interval 0.32 to $0.94)$ and non-melanoma skin cancer $(0.37,0.22$ to 0.62$)$ and an increased risk of death $(1.38,1.14$ to 1.66$)$ (table $4 \Downarrow$ ). The probability of survival at two years was $95.3 \%$ (95\% confidence interval $94.4 \%$ to $96.1 \%$ ) for those receiving sirolimus compared with $96.7 \%$ (95.7\% to $97.5 \%$ ) for those in the control group.
The two year cumulative incidence of cancer was $4.7 \%$ (3.8\% to $5.7 \%$ ) and $7.5 \%$ (6.0\% to $9.4 \%)$, respectively. The two year cumulative incidence of non-melanoma skin cancer was $2.4 \%$ ( $1.8 \%$ to $3.2 \%$ ) and $5.0 \%$ (3.8\% to $6.5 \%)$, respectively.

\section{Discussion Principal findings}

This study included individual patient level data from 21 randomized trials comparing sirolimus with other immunosuppressive regimens in 5876 recipients of kidney transplant. There was a $40 \%$ reduction in the risk of malignancy and a $56 \%$ reduction in risk of non-melanoma skin cancer for those randomized to sirolimus. Patients in conversion trials showed a significant reduction in non-melanoma skin cancer as well as other cancers. In contrast, sirolimus patients enrolled in de novo immunosuppression trials did not have a reduced risk of malignancy or non-melanoma skin cancer principally because of a non- significant increase in the risk of other types of cancer. More importantly, despite the reduction in risk of cancer seen in some patients, use of sirolimus was associated with a significantly increased risk of death in both conversion and de novo trial subgroups.

Although a reduction in cancer was seen in patients converted to sirolimus, the increased risk of death highlights a concerning consequence of this strategy that was not evident from previous trials. By pooling individual patient data, we were able to perform survival analyses, not just on occurrence of cancer but also on overall survival of patients. Our findings help to clarify the benefits and harms associated with sirolimus use regarding reduction of malignancy after kidney transplantation. For patients undergoing transplantation, the use of sirolimus as an initial immunosuppressant cannot be recommended given the lack of efficacy and increased risk of death. In contrast, conversion of patients to sirolimus might reduce the risk of cancer but at the increased cost of death. For every 1000 patients converted to sirolimus and treated for two years, there would be 95 fewer cases of non-melanoma skin cancer and 23 fewer cases of other cancers, but 10 additional deaths. As we observed no reduction in mortality related to cancer, the trade-off of fewer cancers with an increased mortality does not seem justified. Perhaps a subset of patients at greatest risk of cancer might benefit from this strategy, but this would be difficult to predict at the individual level and would require further investigation.

We evaluated several clinically important subgroups. Overall, results were consistent with the main findings that included all available trials and participants. Not surprisingly, there were a few notable differences. For those who received induction therapy, sirolimus use seemed to have no benefit with respect to cancer reduction and was associated with a significant increase risk of death. In patients who did not receive induction therapy, however, there was a significant reduction in cancer with no effect on mortality in those treated with sirolimus (adjusted hazard ratio $1.02,95 \%$ confidence interval 0.77 to 1.34). For recipients of a transplant from a deceased donor, sirolimus was associated with a reduction in cancer and an increased risk of death similar to the overall findings. For recipients from living donors, however, sirolimus had a null effect with respect to mortality $(1.00,0.56$ to 1.78$)$. Unlike the trial level subgroups (for example, de novo or conversion) patients were not randomized to treatment groups based on induction therapy or donor type. This is an important distinction as these subgroups might be imbalanced with respect to other factors that could influence occurrence of cancer or death. While 
interesting clinically, the findings from these subgroup analyses should be interpreted cautiously.

We could not identify the mechanism of cancer reduction in our analysis. The greatest effect was seen in patients converted to sirolimus from another immunosuppressant. Most of these patients were converted from a calcineurin inhibitor, with the remainder being switched from mycophenolate mofetil or azathioprine. We did not have patient level data on drug concentration to assess overall drug exposure, but we did see a consistent effect on cancer reduction regardless of whether the trial used high or low doses of sirolimus. Whether the reduction in malignancy was because of an overall reduction in immunosuppression, removal of oncogenic immunosuppression (such as cyclosporine), or a combination of these factors remains unknown. ${ }^{41}$

The excess risk of death associated with sirolimus could not be directly explained by our data. We found an increase in both cardiovascular deaths and deaths related to infection, the two most common causes of death in patients with kidney transplantation. ${ }^{7}$ Over-immunosuppression with sirolimus could have contributed to the increase in infection related mortality, but this could not be confirmed without data on drug concentration. We did, however, see a significantly increased risk of death in the subgroup of trials that used high dose sirolimus. Overall, net immunosuppression is increased in those who experience acute rejection, given the need for additional corticosteroids or more potent anti-lymphocyte drugs, or both. Patients treated with sirolimus could have had higher net immunosuppression because of the increased risk of acute rejection seen in some of the included trials. ${ }^{20-23}$ This in turn could have contributed to increased infection related mortality. Sirolimus is known to have side effects that are associated with an increased risk of cardiovascular disease, including anemia, ${ }^{20-37}$ proteinuria, ${ }^{37-43}$ hyperglycemia, ${ }^{3744}$ and hyperlipidemia. ${ }^{20-45}$ Although we did not have relevant individual patient level data, an increase in these known cardiac risk factors might have contributed to the increase in cardiovascular death seen in our analysis. It is important to note that death-censored graft survival was not significantly different between the sirolimus and control patients in our analysis. Thus, the increased risk of death was not simply because of more deaths in patients who received dialysis after graft failure, which has been well documented in people with kidney transplant. ${ }^{46} 47$

\section{Comparison with other studies}

The results of this pooled analysis differ from those in previous meta-analyses. Webster and colleagues observed a non-significant trend towards less malignancy and death. ${ }^{17}$ The discrepant findings are probably because of differences in sample size and methods. The Webster review had outcome data (dichotomized as yes/no without respect to timing after transplantation) on only 447 patients for malignancy and 631 patients for survival. We had data (including event dates) on 5876 patients, which permitted survival analysis and increased our statistical power to detect a difference. A second meta-analysis ${ }^{48}$ examining calcineurin inhibitor sparing regimens, which included a subgroup of trials involving sirolimus, did not show any significant effect on patient survival and did not report malignancy. Other sirolimus meta-analyses have been published focusing on dyslipidemia ${ }^{45}$ and wound complications, ${ }^{49}$ but neither reported on cancer or survival. ${ }^{45} 49$ The increased mortality observed in this analysis is consistent with previous non-randomized studies. ${ }^{50-52}$ Isakova and colleagues compared three groups: mTOR inhibitor (99\% sirolimus) without calcineurin inhibitor ( $\mathrm{n}=3237)$, mTOR inhibitor plus a calcineurin inhibitor $(\mathrm{n}=10510)$ and calcineurin inhibitor without mTOR inhibitor $(n=125623) .{ }^{52}$ Compared with the calcineurin inhibitor group, the mTOR inhibitor without calcineurin inhibitor group had a $25 \%$ increased risk of death and the mTOR inhibitor plus calcineurin inhibitor group had a $13 \%$ increased risk of death. The causes of death and malignancy data were not reported. ${ }^{52}$ In another analysis, immunosuppressive regimens containing sirolimus had a risk of death that was 1.33-fold to 1.75 -fold higher compared with other agents. ${ }^{50}$

\section{Strengths and limitations of study}

Our analysis does have some limitations. First, we did not have access to patient level data from all randomized trials of sirolimus in kidney transplantation. We performed an extensive literature search to identify all relevant trials, but the corresponding authors did not respond or agree to share data in all cases. Nonetheless, we were still able to pool individual patient level data on the largest number of randomized patients treated with sirolimus to date. In addition, we pooled the risk of cancer and death from all eligible trials, not just those from which we had individual patient level data. In this meta-analysis of aggregate data, the results were similar to our original analysis and remained significant for the outcomes of any cancer and non-melanoma skin cancer. There was a trend towards an increased risk of death in the aggregate analysis (relative risk $1.21,95 \%$ confidence interval 0.97 to 1.51 ) that became significant when the analysis was restricted to studies with a longer duration of follow-up $(1.42,1.03$ to 1.96$)$. Second, the primary outcome was cancer in only one of the included trials. Given the known association between immunosuppression and malignancy, however, this adverse event is closely monitored in transplantation trials and thus is not likely to be missed during trial follow-up visits. In this analysis, cancer occurred in $4.1 \%$ of patients, which is similar to what has been observed in other transplant trials. ${ }^{53-55}$ Third, there was clinical heterogeneity in the trials included in this analysis, as there often is in systematic reviews. Various immunosuppressive regimens were used in the trials, but all control patients received a calcineurin inhibitor (cyclosporine or tacrolimus), which remains the standard of care in most transplant programs. The trials also included patients with varying risk of malignancy; some studies mandated a history of cancer for inclusion, some excluded those with any cancer, while others excluded only those with a history of non-skin cancer. While these study differences added to the heterogeneity of the analysis, they enhanced the generalizability of our findings to a wider range of patients with different risks of cancer. Fourth, included trials needed a minimum follow-up of three months. While there is no accepted standard for time needed to develop cancer after transplantation, we thought that extending this to six or 12 months might exclude several trials with important and contributable data. Fifth, this study was funded by the manufacturer of sirolimus, which does open the possibility of bias. Although the funding company was allowed to review the manuscript before submission, the concept, design, analysis, interpretation, and writing of this work was done solely by the investigators. Finally, we limited our analysis to randomized trials only. Generalizability of our finding could be improved by the inclusion of non-randomized designs such as cohort and case-control studies. The inclusion of such studies, however, increases the risk of bias and heterogeneity that could make data interpretation more difficult. ${ }^{56}$ 


\section{Conclusions}

In conclusion, sirolimus use was associated with a reduction in the risk of malignancy and non-melanoma skin cancer in patients with kidney transplants. The benefit seemed most pronounced when patients were converted from an established immunosuppressive regimen to sirolimus, with fewer cancers overall as well as fewer non-melanoma skin cancers and other types of cancer. Given the increased risk of mortality, however, the use of this drug does not seem warranted for most patients with kidney transplant. Further research is needed to determine if different populations, such as those at high risk of cancer, might benefit from treatment with sirolimus after kidney transplantation.

We thank Lisa Wilcox (Medical Advisor, Pfizer, Canada), who helped coordinate data retrieval from the Wyeth/Pfizer trials.

Contributors: GAK and DAF initiated and designed the study. GAK obtained funding. MBK, $A B, C D B$, and RD screened and identified trials of inclusion, abstracted data, and prepared the databases. GAK, MBK, $\mathrm{RM}$, and DAF performed the analyses. MBK and GAK drafted the manuscript. RB, CB, TDBH, HE, CRF, JF, LG, MG, DG, JG, MG, AAM, RS, EHS, HTS, SV, and CW provided trial data. All authors made critical comments, suggestions and revisions to earlier drafts. All authors interpreted results and approved the final version of the paper. GAK is guarantor.

Funding: This study was funded by an investigator grant from Pfizer (previously Wyeth Pharmaceuticals). Pfizer did not have any role in the study concept, design, data analysis, or writing of the manuscript. Representatives from Pfizer were given a copy of the manuscript to review before submission for publication.

Competing interests: All authors have completed the ICMJE uniform disclosure form at www.icmje.org/coi_disclosure.pdf and declare the following: GAK has received honorariums and/or research support from Astellas Canada, Pfizer Canada, Roche Canada, and Novartis Canada. $A B, C D B$, and MBK had partial salary support from Roche Canada. $C B$ has received personal fees from Hoffmann-La Roche unrelated to this submitted work; TDBH received a grant from Wyeth for an investigator initiated trial that was included as part of this submitted work; TDBH has received personal fees from Astellas Canada for advisory board activities unrelated to this submitted work; HTS has received personal fees and research grant support from Pfizer unrelated to this submitted work; CW received a grant from Wyeth for a trial that was included as part of this submitted work; CW has received personal fees from Wyeth for advisory board activities and lectures.

Ethical approval: The study was approved by the Ottawa Hospital research ethics board (protocol \#20120638).

Transparency: GAK affirms that this paper is an honest, accurate and transparent account of the study being reported; no important aspects of the study have been omitted.

Data sharing: We do not have authorization to release individual patient level data from the trials in this analysis.

1 Wolfe RA, Ashby VB, Milford EL, Ojo AO, Ettenger RE, Agodoa LYC, et al. Comparison of mortality in all patients on dialysis, patients on dialysis awaiting transplantation, and recipients of a first cadaveric transplant. N Engl J Med 1999;341:1725-30.

2 Laupacis A, Keown P, Pus N, Krueger H, Ferguson B, Wong C, Muirhead N. A study of the quality of life and cost-utility of renal transplantation. Kidney Int 1996;50:235-42.

3 US Renal Data System. USRDS 2013 Annual data report: atlas of chronic kidney disease and end-stage renal disease in the United States, National Institutes of Health, National Institute of Diabetes and Digestive and Kidney Diseases, 2013.

4 WHO-ONT collaboration. Global observatory on donation and transplantation. www. transplant-observatory.org/Pages/home.aspx

5 Vajdic CM, McDonald SP, McCredie MR, van Leeuwen MT, Stewart JH, Law M, et al. Cancer incidence before and after kidney transplantation. JAMA 2006;296:2823-31.

6 Krynitz B, Edgren G, Lindelöf B, Baecklund E, Brattström C, Wilczek H, Smedby KE. Risk of skin cancer and other malignancies in kidney, liver, heart and lung transplant recipients 1970 to 2008-a Swedish population-based study. Int J Cancer 2013;132:1429-38.

7 US Renal Data System. USRDS 2012 annual data report: atlas of chronic kidney disease and end-stage renal disease in the United States. National Institutes of Health. National Institute of Diabetes and Digestive and Kidney Diseases, 2012.
8 ANZDATA Registry Report 2010, Australia and New Zealand Dialysis and Transplant Registry, Adelaide, South Australia. Australia and New Zealand Dialysis and Transplant Registry, 2010.

9 Asch WS, Bia MJ. Oncologic issues and kidney transplantation: a review of frequency, mortality, and screening. Adv Chronic Kidney Dis 2014;21:106-13.

10 Mackenzie KA, Wells JE, Lynn KL, Simcock JW, Robinson BA, Roake JA, et al. First and subsequent nonmelanoma skin cancers: incidence and predictors in a population of New Zealand renal transplant recipients. Nephrol Dial Transplant 2010;25:300-6.

11 Wisgerhof HC, Edelbroek JR, de Fijter JW, Haasnoot GW, Claas FH, Willemze R, et al. Subsequent squamous- and basal-cell carcinomas in kidney-transplant recipients after the first skin cancer: cumulative incidence and risk factors. Transplantation 2010;89:1231-8.

12 Zavos G, Karidis NP, Tsourouflis G, Bokos J, Diles K, Sotirchos G, et al. Nonmelanoma skin cancer after renal transplantation: a single-center experience in 1736 transplantations. Int J Dermatol 2011;12:1496-500.

13 Khokhar NZ, Altman JK, Platanias LC. Emerging roles for mammalian target of rapamycin inhibitors in the treatment of solid tumors and hematological malignancies. Curr Opin Oncol 2011;23:578-86.

14 Kapoor A. Malignancy in kidney transplant recipients. Drugs 2008;68(suppl 1):11-9.

15 Campistol JM, Albanell J, Arns W, Boletis I, Dantal J, de Fijter JW, et al. Use of proliferation signal inhibitors in the management of post-transplant malignancies--clinical guidance. Nephrol Dial Transplant 2007;22(suppl 1):i36-41.

16 Kauffman H, Cherikh W, Cheng Y, Hanto D, Kahan BD. Maintenance immunosuppression with target-of-rapamycin inhibitors is associated with a reduced incidence of de novo malignancies. Transplantation 2005;80:883-9.

17 Webster AC, Lee VW, Chapman JR, Craig JC. Target of rapamycin inhibitors (sirolimus and everolimus) for primary immunosuppression of kidney transplant recipients: a systematic review and meta-analysis of randomized trials. Transplantation 2006;81:1234-48.

18 Campbell S, Walker R, Tai SS, Jiang Q, Russ G. Randomized controlled trial of sirolimus for renal transplant recipients at high risk for nonmelanoma skin cancer. Am J Transplant 2012;12:1146-56.

19 Euvrard S, Morelon E, Rostaing L, Goffin E, Brocard A, Tromme I, et al. Sirolimus and secondary skin-cancer prevention in kidney transplantation. N Engl J Med 2012;26:329-39.

20 Ekberg H, Tedesco-Silva H, Demirbas A, Vítko S, Nashan B, Gürkan A, et al. Reduced exposure to calcineurin inhibitors in renal transplantation. N Engl J Med 2007;357:2562-75.

21 Altman DG, Andersen PK. Calculating the number needed to treat for trials where the outcome is time to an event. BMJ 1999;319:1492-5.

22 Flechner S, Glyda M, Cockfield S, Grinyó J, Legendre Ch, Russ G, et al. The ORION study: comparison of two sirolimus-based regimens versus tacrolimus and mycophenolate mofetil in renal allograft recipients. Am J Transplant 2011;11:1633-44.

23 Flechner SM, Gurkan A, Hartmann A, Legendre CM, Russ GR, Campistol JM, et al. A randomized, open-label study of sirolimus versus cyclosporine in primary de novo renal allograft recipients. Transplantation 2013;95:1233-41.

24 Kreis H, Cisterne JM, Land W, Wramner L, Squifflet JP, Abramowicz D, et al. Sirolimus in association with mycophenolate mofetil induction for the prevention of acute graft rejection in renal allograft recipients. Transplantation 2000;69:1252-60.

25 MacDonald AS, RAPAMUNE Global Study Group. A worldwide, phase III, randomized, controlled, safety and efficacy study of a sirolimus/cyclosporine regimen for prevention of acute rejection in recipients of primary mismatched renal allografts. Transplantation 2001;71:271-80.

26 Barsoum RS, Morsy AA, Iskander IR, Morgan MM, Fayad TM, Atalla NT, et al. The Cairo kidney center protocol for rapamycin-based sequential immunosuppression in kidney transplant recipients: 2-year outcomes. Exp Clin Transplant 2007;5:649-57.

27 Blydt-Hansen TD, Gibson IW, Birk PE. Histological progression of chronic renal allograft injury comparing sirolimus and mycophenolate mofetil-based protocols. A single-center, prospective, randomized, controlled study. Pediatr Transplant 2010;14:909-18.

28 Gallon L, Perico N, Dimitrov BD, Winoto J, Remuzzi G, Leventhal J, et al. Long-term renal allograft function on a tacrolimus-based, pred-free maintenance immunosuppression comparing sirolimus vs. MMF. Am J Transplant 2006;6:1617-23.

29 Gelens MACJ, Christiaans MHL, Van Heurn ELW, Berg-Loonen EPM, Peutz-Kootstra $\mathrm{CJ}$, Van Hooff JP. High rejection rate during calcineurin inhibitor-free and early steroid withdrawal immunosuppression in renal transplantation. Transplantation 2006;82:1221-3.

30 Glotz D, Charpentier B, Abramovicz D, Lang P, Rostaing L, Rifle G, et al. Thymoglobulin induction and sirolimus versus tacrolimus in kidney transplant recipients receiving mycophenolate mofetil and steroids. Transplantation 2010;89:1511-7.

31 Guba M, Pratschke J, Hugo C, Krämer BK, Nohr-Westphal C, Brockmann J, et al. Renal function, efficacy, and safety of sirolimus and mycophenolate mofetil after short-term calcineurin inhibitor-based quadruple therapy in de novo renal transplant patients: one-year analysis of a randomized multicenter trial. Transplantation 2010;90:175-83.

32 Groth CG, Backman L, Morales JM, Calne R, Kreis H, Lang P, et al. Sirolimus (rapamycin)-based therapy in human renal transplantation: Similar efficacy and different toxicity compared with cyclosporine. Transplantation 1999;67:1036-42.

33 Kahan BD, Julian BA, Pescovitz MD, Vanrenterghem Y, Neylan J. Sirolimus reduces the incidence of acute rejection episodes despite lower cyclosporine doses in caucasian recipients of mismatched primary renal allografts: a phase II trial. Transplantation 1999;68:1526-32.

34 Kahan BD for the Rapamune US Study Group. Efficacy of sirolimus compared with azathioprine for reduction of acute renal allograft rejection: a randomised multicentre study. Lancet 2000;356:194-202.

35 Machado PGP, Felipe CR, Hanzawa NM, Park SI, Garcia R, Alfieri F, et al. An open-label randomized trial of the safety and efficacy of sirolimus vs. azathioprine in living related renal allograft recipients receiving cyclosporine and prednisone combination. Clin Transplant 2004;18:28-38.

36 Salgo R, Gossmann J, Schofer H, Kachel HG, Kuck J, Geiger H, et al. Switch to a sirolimus-based immunosuppression in long-term renal transplant recipients: reduced rate of (pre) malignancies and nonmelanoma skin cancer in a prospective, randomized, assessor-blinded, controlled clinical trial. Am J Transplant 2010;10:1385-93.

37 Schena FP, Pascoe MD, Alberu J, del Carmen Rial M, Oberbauer R, Brennan DC, et al. Conversion from calcineurin inhibitors to sirolimus maintenance therapy in renal allograft recipients: 24-month efficacy and safety results from the CONVERT trial. Transplantation 2009;87:233-42.

38 Sampaio EL, Pinheiro-machado PG, Garcia R, Felipe CR, Park SI, Casarini DE, et al. Mycophenolate mofetil vs. sirolimus in kidney transplant recipients receiving tacrolimus-based immunosuppressive regimen. Clin Transplant 2008;22:141-9. 


\section{What is already known on this topic}

The incidence of most malignancies increases after kidney transplantation, especially non-melanoma skin cancer Observational studies and some randomized trials suggest that the immunosuppressive drug sirolimus could reduce the incidence of cancers

A previous meta-analysis found no significant association between sirolimus use and malignancy or death

\section{What this study adds}

An individual patient level meta-analysis of nearly 6000 kidney transplant recipients found that sirolimus significantly reduced malignancy and non-melanoma skin cancer but increased death

Sirolimus can reduce the risk of cancer but the use of this drug does not seem warranted for most patients with kidney transplant given the excess risk of mortality

39 Vitko S, Wlodarczyk Z, Kyllonen L, Czajkowski Z, Margreiter R, Backman L, et al. Tacrolimus combined with two different dosages of sirolimus in kidney transplantation: results of a multicenter study. Am J Transplant 2006;6:531-8.

40 Watson CJE, Firth J, Williams PF, Bradley JR, Pritchard N, Chaudhry A, et al. A randomized controlled trial of late conversion from $\mathrm{CNI}$-based to sirolimus-based immunosuppression following renal transplantation. Am J Transplant 2005:5:2496-503.

41 Halleck F, Budde K. Transplantation: sirolimus for secondary SCC prevention in renal transplantation. Nat Rev Nephrol 2012;8:687

42 Letavernier E, Legendre CM. mToR inhibitors-induced proteinuria: mechanisms, significance, and management. Tranplant Rev 2008;22:125-30.

43 Halimi JM. Low-grade proteinuria and microalbuminuria in renal transplantation. Transplantation 2013:96:121-30.

44 Johnston O, Rose CL, Webster AC, Gill J. Sirolimus is associated with new-onset diabetes in kidney transplant recipients. J Am Soc Nephrol 2008;19:1411-8.

45 Kasiske B, de Mattos A, Flechner S, Gallon L, Meier-Kriesche HU, Weir MR, et al. Mammalian target of rapamycin inhibitor dyslipidemia in kidney transplant recipients. Am $J$ Transplant 2008;8:1384-92.

46 Knoll G, Muirhead N, Trpeski L, Zhu N, Badovinac K. Patient survival following renal transplant failure in Canada. Am J Transplant 2005;5:1719-24.

47 Kaplan B, Meier-Kriesche HU. Death after graft loss: an important late study endpoint in kidney transplantation. Am J Transplant 2002;2:970-4.

48 Sharif A, Shabir S, Chand S, Cockwell P, Ball S, Borrows R. Meta-analysis of calcineurin-inhibitor-sparing regimens in kidney transplantation. J Am Soc Nephrol 2011;22:2107-18.

49 Pengel L, Liu H, Morris P. Do wound complications or lymphoceles occur more often in solid organ transplant recipients on $m$ TOR inhibitors? A systematic review of randomized controlled trials. Transplant Int 2011;24:1216-30.

50 Srinivas TR, Schold JD, Guerra G, Eagan A, Bucci CM, Meier-Kriesche HU. Mycophenolate mofetil/sirolimus compared to other common immunosuppressive regimens in kidney transplantation. Am J Transplant 2007;7:586-94.
51 Cortazar F, Molnar MZ, Isakova T, Czira ME, Kovesdy CP, Roth D, et al. Clinical outcomes in kidney transplant recipients receiving long-term therapy with inhibitors of the mammalian target of rapamycin. Am J Transplant 2012;12:379-87.

52 Isakova T, Xie H, Messinger S, Cortazar F, Scialla JJ, Guerra G, et al. Inhibitors of mTOR and risks of allograft failure and mortality in kidney transplantation. Am J Transplant 2013;13:100-10.

53 Russ GR, Tedesco-Silva H, Kuypers D, Cohney S, Langer RM, Witzke O, et al. Efficacy of sotrastaurin plus tacrolimus after de novo kidney transplantation: randomized, phase II trial results. Am J Transplant 2013;13:1746-56.

54 Kramer BK, Klinger M, Vitko S, Glyda M, Midtvedt K, Stefoni S, et al. Tacrolimus-based, steroid-free regimens in renal transplantation: 3-year follow-up of the ATLAS trial. Transplantation 2012;94:492-8.

55 Vincenti F, Tedesco-Silva H, Busque S, O'Connell P, Friedewald J, Cibrik D, et al. Randomized phase $2 b$ trial of tofacitinib $(\mathrm{CP}-690,550)$ in de novo kidney transplant patients: efficacy, renal function and safety at 1 year. Am J Transplant 2012;12:2446-56.

56 Reeves BC, Deeks JJ, Higgins JPT, Wells GA. Including non-randomized studies. In: Higgins JPT, Green S, eds. Cochrane Handbook for Systematic Reviews of Interventions. Wiley-Blackwell, 2008:391-432.

\section{Accepted: 20 October 2014}

\section{Cite this as: BMJ 2014;349:g6679}

This is an Open Access article distributed in accordance with the Creative Commons Attribution Non Commercial (CC BY-NC 4.0) license, which permits others to distribute, remix, adapt, build upon this work non-commercially, and license their derivative works on different terms, provided the original work is properly cited and the use is non-commercial. See: http://creativecommons.org/licenses/by-nc/4.0/. 


\section{Tables}

Table 1 | Baseline characteristics of patients with kidney transplant according to immunosuppressive treatment group. Figures are numbers (percentage) of patients unless stated otherwise

\begin{tabular}{|c|c|c|c|}
\hline Variable & Sirolimus group $(n=3276)$ & Control group $(n=2600)$ & All $(n=5876)$ \\
\hline \multicolumn{4}{|l|}{ Age (years): } \\
\hline Mean (SD) & $45.4(13.6)$ & $45.4(13.8)$ & $45.4(13.7)$ \\
\hline Median (IQR) & $47.0(35.0-56.0)$ & $46.4(35.0-56.0)$ & $46.7(35.0-56.0)$ \\
\hline \multicolumn{4}{|l|}{ Sex: } \\
\hline Female & 1055 (32.2) & $883(34.0)$ & $1938(33.0)$ \\
\hline Male & $2221(67.8)$ & $1717(66.0)$ & $3938(67.0)$ \\
\hline \multicolumn{4}{|l|}{ Previous transplant: } \\
\hline No & 3159 (96.4) & $2496(96.0)$ & 5655 (96.2) \\
\hline Yes & $117(3.6)$ & $103(4.0)$ & $220(3.7)$ \\
\hline Unknown & $0(0)$ & $1(0)$ & $1(0)$ \\
\hline \multicolumn{4}{|l|}{ Region of enrolment: } \\
\hline Europe & $1206(36.8)$ & $1451(55.8)$ & $2657(45.2)$ \\
\hline North America & $1380(42.1)$ & $524(20.2)$ & $1904(32.4)$ \\
\hline South America & $364(11.1)$ & $376(14.5)$ & $740(12.6)$ \\
\hline Oceania & $191(5.8)$ & $136(5.2)$ & $327(5.6)$ \\
\hline Africa & $102(3.1)$ & $52(2.0)$ & $154(2.6)$ \\
\hline Asia & $33(1.0)$ & $61(2.4)$ & $94(1.6)$ \\
\hline \multicolumn{4}{|l|}{ Trial type: } \\
\hline De novo* & $2558(78.1)$ & $2159(83.0)$ & $4717(80.3)$ \\
\hline Conversion† & $718(21.9)$ & $441(17.0)$ & $1159(19.7)$ \\
\hline \multicolumn{4}{|l|}{ Comparator drugs(s): } \\
\hline Anti-metaboliteł & - & $189(7.3)$ & - \\
\hline Calcineurin inhibitor§ & - & $1914(73.6)$ & - \\
\hline Anti-metabolite and calcineurin inhibitor & - & $341(13.1)$ & - \\
\hline Placebo & - & $156(6.0)$ & - \\
\hline
\end{tabular}

IQR=interquartile range.

*Sirolimus or control agent given as initial immunosuppression.

†Sirolimus replacing another immunosuppressive agent.

$\ddagger$ Azathioprine or mycophenolate mofetil.

$\S$ Tacrolimus or cyclosporine. 
Table 2| Hazard ratios (95\% confidence interval) ${ }^{\star}$ and $\mathrm{P}$ values for risk of cancer and death in patients with kidney transplant according to immunosuppressive treatment group†

\begin{tabular}{|c|c|c|c|c|}
\hline Variable & Any cancer & Non-melanoma skin cancer & Other cancer & Death \\
\hline Control group & 1.00 & 1.00 & 1.00 & 1.00 \\
\hline Sirolimus group & 0.60 (0.39 to 0.93 ), 0.02 & $0.44(0.30$ to 0.63$),<0.001$ & 1.05 (0.57 to 1.94$), 0.88$ & $1.43(1.21$ to 1.71$),<0.001$ \\
\hline Age $\ddagger$ & 1.06 (1.04 to 1.09$),<0.001$ & 1.08 (1.06 to 1.10$),<0.001$ & 1.05 (1.02 to 1.08$),<0.001$ & 1.07 (1.05 to 1.08$),<0.001$ \\
\hline Female sex & 0.80 (0.66 to 0.97 ), 0.03 & 0.79 (0.57 to 1.10$), 0.16$ & 0.79 (0.55 to 1.13$), 0.19$ & 1.00 (0.80 to 1.25$), 0.96$ \\
\hline $\begin{array}{l}\text { Time after transplantation } \\
\text { to randomization } \ddagger \\
\end{array}$ & 1.11 (1.08 to 1.15$),<0.001$ & $1.13(1.08$ to 1.20$),<0.001$ & 1.02 (0.98 to 1.07$), 0.33$ & 0.93 (0.88 to 0.99$), 0.02$ \\
\hline \multicolumn{5}{|l|}{ Location: } \\
\hline North America & 1.00 & 1.00 & 1.00 & 1.00 \\
\hline Africa & $0.31(0.23$ to 0.41$),<0.001$ & 0.30 (0.06 to 1.62$), 0.16$ & 0.30 (0.08 to 1.12 ), 0.07 & 1.42 (0.91 to 2.20$), 0.12$ \\
\hline Asia & 0.87 (0.58 to 1.30$), 0.49$ & $0.33(0.04-3.05), 0.33$ & 1.52 (0.84 to 2.78$), 0.16$ & 1.74 (1.01 to 3.01$), 0.046$ \\
\hline Europe & $0.58(0.44$ to 0.76$),<0.001$ & 0.44 (0.23 to 0.84$), 0.01$ & 0.87 (0.58 to 1.30$), 0.49$ & 1.23 (0.89 to 1.71$), 0.21$ \\
\hline Oceania & 3.68 (2.21 to 6.13$),<0.001$ & 5.13 (2.64 to 9.98$),<0.001$ & 1.76 (1.13 to 2.73 ), 0.01 & 1.16 (0.65 to 2.07$), 0.62$ \\
\hline South America & 0.59 (0.42 to 0.85$), 0.004$ & 0.74 (0.51 to 1.08$), 0.12$ & 0.41 (0.24 to 0.70$), 0.001$ & 1.67 (1.18 to 2.37$), 0.004$ \\
\hline
\end{tabular}

${ }^{*}$ Calculated with Cox proportional hazards model. All models adjusted for trial, age, sex, geographic location of participant, and time after transplantation to randomization.

†Three patients had both non-melanoma skin cancer and another cancer recorded on same date; each diagnosis was counted in relevant category. ‡Per year. 
Table 3| Aggregate relative risk of any cancer, non-melanoma skin cancer, and death in patients with kidney transplant from trials with individual patient level data and trials with aggregate data*

\begin{tabular}{|c|c|c|c|c|c|c|c|c|c|c|c|c|}
\hline \multirow[b]{2}{*}{ Trial type } & \multicolumn{4}{|c|}{ Any cancer } & \multicolumn{4}{|c|}{ Non-melanoma skin cancer } & \multicolumn{4}{|c|}{ Death } \\
\hline & Trials & Patients & Events & $\begin{array}{l}\text { Risk ratio }(95 \% \\
\text { Cl) }\end{array}$ & Trials & Patients & Events & $\begin{array}{l}\text { Risk ratio }(95 \% \\
\text { Cl) }\end{array}$ & Trials & Patients & Events & $\begin{array}{c}\text { Risk ratio }(95 \% \\
\text { Cl) }\end{array}$ \\
\hline $\begin{array}{l}\text { Individual } \\
\text { patient level } \\
\text { data }\end{array}$ & 21 & 5876 & 243 & $0.69(0.51$ to 0.94$)$ & 21 & 5876 & 150 & $0.48(0.35$ to 0.66$)$ & 21 & 5876 & 202 & $1.28(0.92$ to 1.76$)$ \\
\hline $\begin{array}{l}\text { Aggregate } \\
\text { data }\end{array}$ & 16 & 3521 & 116 & 0.48 (0.29 to 0.79$)$ & 9 & 2517 & 71 & $0.46(0.30$ to 0.71$)$ & 29 & 4059 & 160 & 1.15 (0.40 to 1.56$)$ \\
\hline Combined & 37 & 9397 & 359 & $0.63(0.48$ to 0.81$)$ & 30 & 8393 & 221 & $0.47(0.37$ to 0.61$)$ & 50 & 9935 & 362 & $1.21(0.97$ to 1.51$)$ \\
\hline
\end{tabular}

*Unadjusted risk calculated with random effects model. 
Table 4| Hazard ratios (95\% confidence interval) ${ }^{\star}$ and $\mathbf{P}$ values for risk of cancer and death in patients with kidney transplant according to immunosuppressive treatment group with follow-up censored at 24 months

\begin{tabular}{lcccc} 
Variable & Any cancer & Non-melanoma skin cancer & Other cancer & Death \\
Control group & 1.00 & 1.00 & 1.00 & 1.00 \\
\hline Sirolimus group & $0.55(0.32$ to 0.94$), 0.03$ & $0.37(0.22$ to 0.62$),<0.001$ & $1.04(0.51$ to 2.12$), 0.92$ & $1.38(1.14$ to 1.66$),<0.001$ \\
\hline Aget & $1.06(1.04$ to 1.09$),<0.001$ & $1.08(1.06$ to 1.10$),<0.001$ & $1.05(1.02$ to 1.08$), 0.001$ & $1.07(1.05$ to 1.08$),<0.001$ \\
\hline Female sex & $0.79(0.63$ to 0.99$), 0.04$ & $0.77(0.49$ to 1.18$), 0.23$ & $0.78(0.52$ to 1.16$), 0.22$ & $0.98(0.77$ to 1.24$), 0.84$ \\
\hline $\begin{array}{l}\text { Time after transplantation } \\
\text { to randomizationt }\end{array}$ & $1.11(1.08$ to 1.15$),<0.001$ & $1.14(1.08$ to 1.21$),<0.001$ & 1.02 (0.97 to 1.07$), 0.55$ & $0.92(0.86$ to 0.98$), 0.01$ \\
\hline Location: & & & & \\
\hline North America & 1.00 & 1.00 & 1.00 & 1.00 \\
\hline Africa & $0.37(0.19$ to 0.71$), 0.003$ & $0.34(0.04$ to 2.62$), 0.29$ & $0.38(0.17$ to 0.87$), 0.02$ & $1.23(0.73$ to 2.24$), 0.39$ \\
\hline Asia & $0.68(0.43$ to 1.06$), 0.09$ & $-\neq$ & $1.66(1.09$ to 2.52$), 0.02$ & $1.63(0.98$ to 2.69$), 0.06$ \\
\hline Europe & $0.52(0.39$ to 0.68$),<0.001$ & $0.34(0.18$ to 0.63$),<0.001$ & $0.9(0.63$ to 1.28$), 0.56$ & $1.10(0.81$ to 1.49$), 0.53$ \\
\hline Oceania & $3.41(1.99$ to 5.83$),<0.001$ & $4.23(2.12$ to 8.45$),<0.001$ & $1.70(1.01$ to 2.88$), 0.047$ & $1.05(0.52$ to 2.12$), 0.88$ \\
\hline South America & $0.45(0.28$ to 0.73$), 0.001$ & $0.55(0.32$ to 0.95$), 0.03$ & 0.33 (0.12 to 0.89$), 0.03$ & 1.32 (0.75 to 2.34$), 0.34$ \\
\hline
\end{tabular}

${ }^{*}$ Calculated with Cox proportional hazards model. All models adjusted for trial, age, sex, geographic location of participant, and time after transplantation to randomization.

†Per year.

¥Hazard ratio not estimable; too few events. 


\section{Figures}

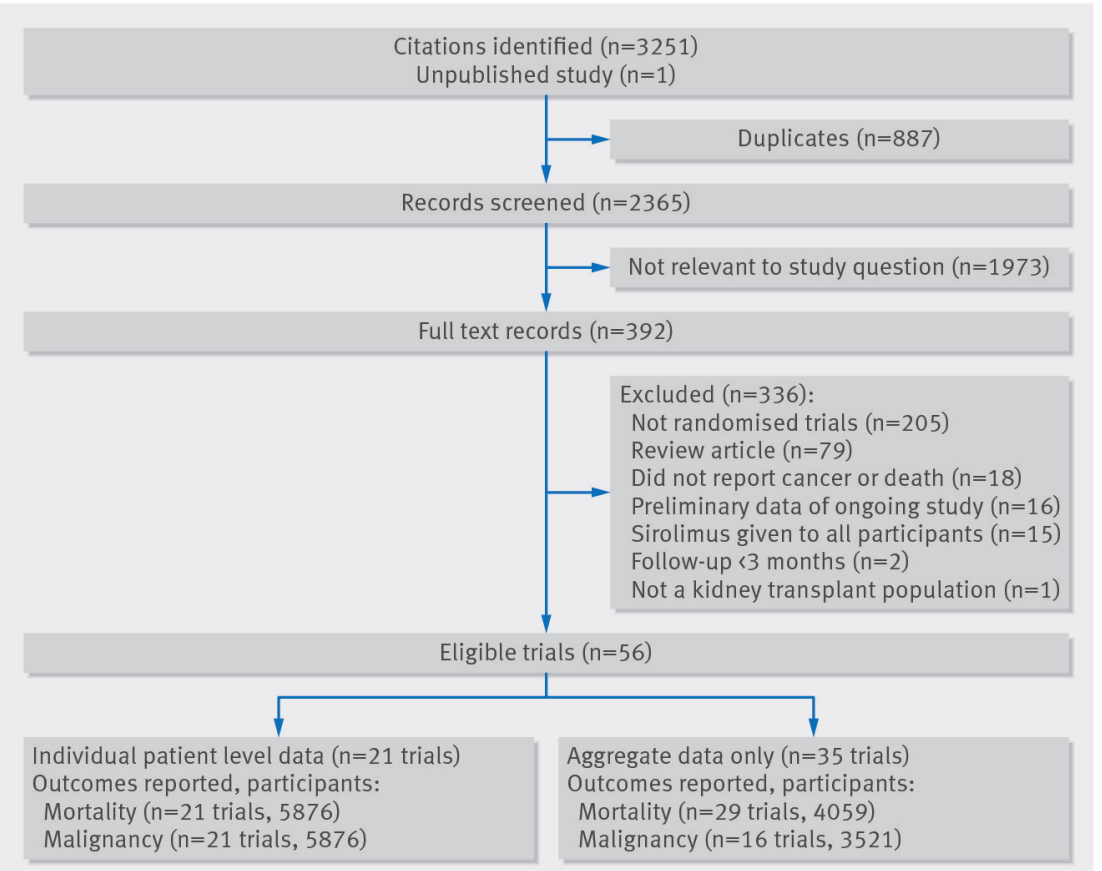

Fig 1 PRISMA flow chart of included trials on effect of sirolimus on malignancy and survival after kidney transplantation

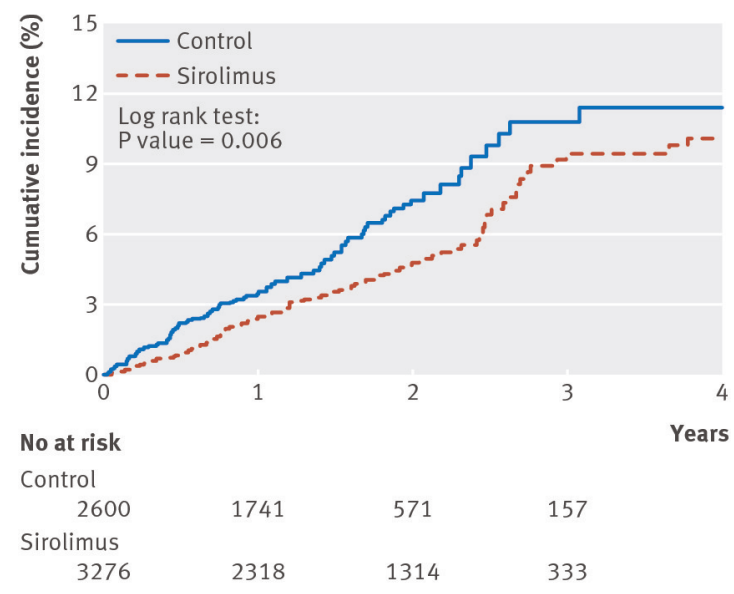

Fig 2 Time to first malignancy in patients with kidney transplant according to immunosuppressive treatment group 


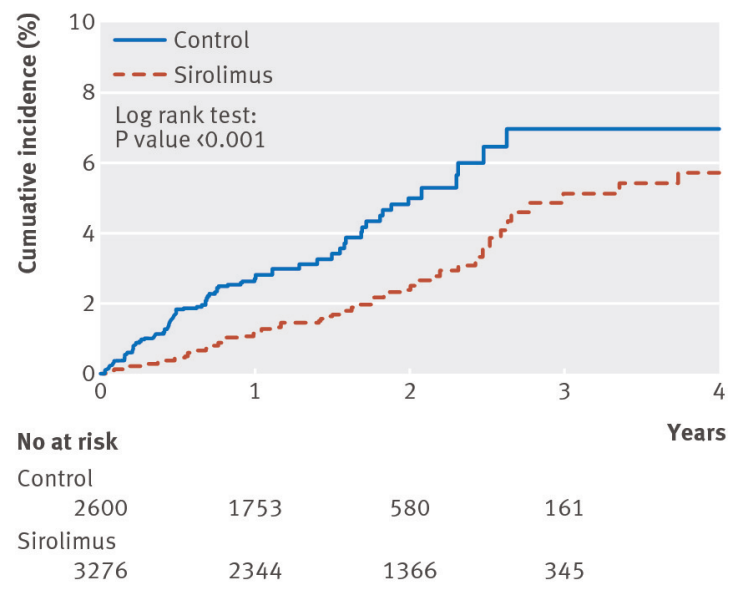

Fig 3 Time to first non-melanoma skin cancer in patients with kidney transplant according to immunosuppressive treatment group

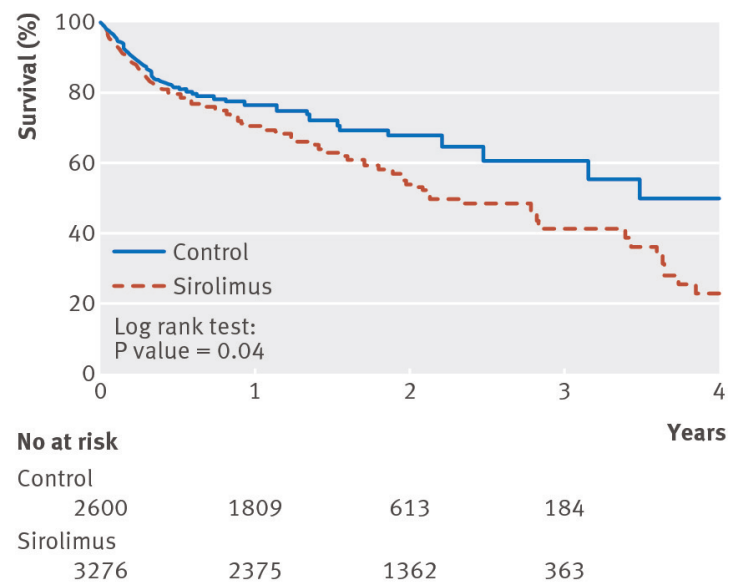

Fig 4 Overall survival in patients with kidney transplant according to immunosuppressive treatment group 


\section{Trials Events Patients}

\section{Any cancer}

All trials

De novo trials

Conversion trials

Low dose sirolimus trials

High dose sirolimus trials

Non-melanoma skin cance

All trials

De novo trials

Conversion trials

Low dose sirolimus trials

High dose sirolimus trials

\section{Other cancer}

All trials

De novo trials

Conversion trials

Low dose sirolimus trials

High dose sirolimus trials

Death

All trials

De novo trials

Conversion trials

Low dose sirolimus trials

High dose sirolimus trials

\section{Hazard ratio} (95\% Cl)

5876

$109 \quad 4717$

$134 \quad 1159$

$76 \quad 2384$

$167 \quad 3492$

150

51

99

59

96

106

61

45

45

$12 \quad 24$

82

202

159

43

43
61

141

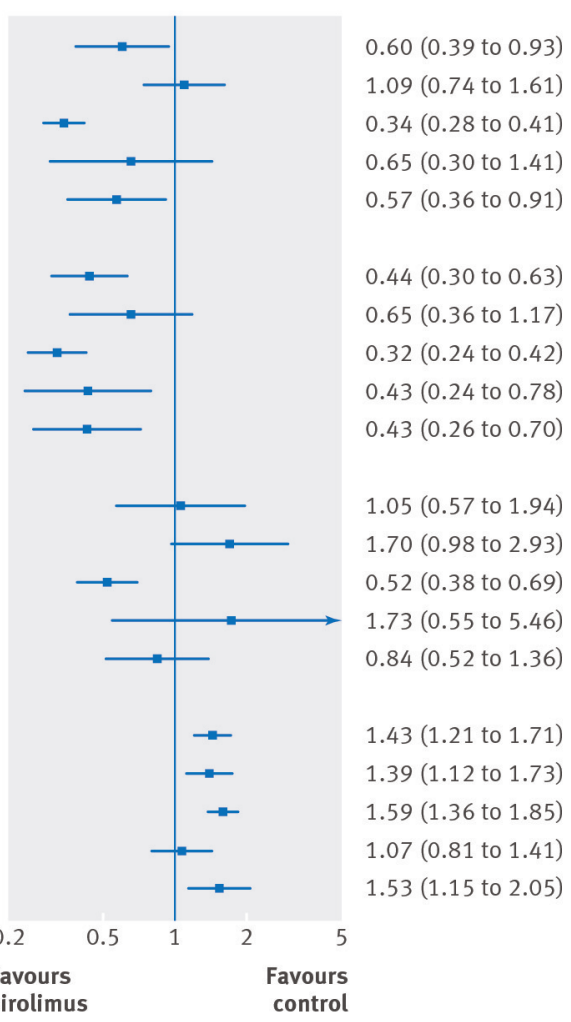

Fig 5 Risk of cancer and death in patients with kidney transplant treated with sirolimus versus control 